\title{
The Approximation of Hermite Interpolation on the Weighted Mean Norm
}

\author{
Xin Wang 1 , Chong $\mathrm{Hu}^{2}$, Xiuxiu $\mathrm{Ma}^{3}$ \\ ${ }^{1}$ Department of Mathematics and Computer, Baoding University, Baoding, China \\ ${ }^{2}$ Institute of Nuclear Technology, China Institute of Atomic Energy, Beijing, China \\ ${ }^{3}$ Institute of Mathematical, North China Electric Power University, Baoding, China \\ Email: wangxincloud@163.com
}

Received 22 July 2015; accepted 11 September 2015; published 14 September 2015

Copyright (C) 2015 by authors and Scientific Research Publishing Inc.

This work is licensed under the Creative Commons Attribution International License (CC BY). http://creativecommons.org/licenses/by/4.0/

cc) (i) Open Access

\section{Abstract}

We research the simultaneous approximation problem of the higher-order Hermite interpolation based on the zeros of the second Chebyshev polynomials under weighted Lp-norm. The estimation is sharp.

\section{Keywords}

Hermite Interpolation Operator, Chebyshev Polynomial, Derivative Approximation

\section{Introduction}

For $0<p<+\infty$ and a non-negative measurable function $u$, the space $L_{u}^{p}$ is defined to be the set of measurable $f$, such that

$$
\|f\|_{p, u}=\left(\int_{-1}^{1}|f(t)|^{p} u(t) \mathrm{d} t\right)^{1 / p}, \quad 0<p<+\infty
$$

is finite. Of course, when $0<p<1,\|\cdot\|_{p, u}$ is not a norm; nevertheless, we keep this notation for convenience. For $u=1$, this is the usual $L^{p}$ space. For $d \in N$, we write $C^{d}$ for the space of functions that have $d$ th continuous derivative on $[-1,1]$.

We introduce a few notations. If $\omega$ is a Jacobi weight function, we write $\omega \in J$. Let $\omega \in J, \omega(x)=\omega^{(\alpha, \beta)}(x)=(1-x)^{\alpha}(1+x)^{\beta}$. The Jacobi polynomials $p_{n}(\omega)$ are orthogonal polynomials with respect to the weight function $\omega$, i.e.

$$
\int_{-1}^{1} p_{n}(\omega, x) p_{m}(\omega, x) \omega(x) \mathrm{d} x=\delta_{n, m}
$$


It is well known that $p_{n}(\omega)$ has $n$ distinct zeros in $(-1,1)$. These zeros are denoted by $x_{k n}(\omega)$ and the following order is assumed:

$$
1>x_{1 n}(\omega)>x_{2 n}(\omega)>\cdots>x_{n n}(\omega)>-1
$$

Later, when we fix $\omega$, we shall write $x_{k n}$ instead of $x_{k n}(\omega)$.

For a given integer $r \geq 0, s \geq 0$ and $m \geq 1$, the Hermite interpolation is defined to be the unique polynomial of degree $N=m n+r+s-1$, denoted by $H_{n, m, r, s}(\omega, f)$, satisfying

$$
\left\{\begin{array}{l}
H_{n, m, r, s}^{(t)}\left(\omega, f, x_{k n}\right)=f^{(t)}\left(x_{k n}\right), 0 \leq t \leq m-1,1 \leq k \leq n ; \\
H_{n, m, r, s}^{(t)}(\omega, f, 1)=f^{(t)}(1), 0 \leq t \leq r-1 ; \\
H_{n, m, r, s}^{(t)}(\omega, f,-1)=f^{(t)}(-1), 0 \leq t \leq s-1
\end{array}\right.
$$

for $f \in C^{M}$, where $M=\max \{m-1, r-1, s-1\}$, if $r=0$ or $s=0$ then we have no interpolation at 1 or -1 . We shall fix the integers $m, r$ and $s$ for the rest of the paper, and omit them from the notations. Thus, for example, we shall write $H_{n}(\omega, f)$ instead of $H_{n, m, r, s}(\omega, f)$. Let

$$
\varphi(x)=\sqrt{1-x^{2}}, \omega_{m}^{(r, s)}:=\left[(1-x)^{\alpha-\frac{2 r}{m}+\frac{1}{2}}(1+x)^{\beta-\frac{2 s}{m}+\frac{1}{2}}\right]^{\frac{m}{2}} .
$$

Vertesi and $\mathrm{Xu}$ [1], Nevai and $\mathrm{Xu}$ [2], and Pottinger considered the simultaneous approximation by Hermite interpolation operators.

We have researched the simultaneous approximation problem of the lower-order Hermite interpolation based on the zeros of Chebyshev polynomials under weighted Lp-norm in references [3]-[5]. We will research the simultaneous approximation problem of the higher-order Hermite interpolation in this article.

Let

$$
X_{n}=\left\{X_{k}=\cos \theta_{k}=\cos \frac{k \pi}{n+1}: 1 \leq k \leq n\right\}
$$

be the zeros of $U_{n}(x)=\frac{\sin (n+1) \theta}{\sin \theta}, x=\cos \theta$, the $n$th degree Chebyshev polynomial of the second kind. For $f \in C_{[-1,1]}^{2}$, let $H_{n}(f, x)$ be the polynomial of degree at most $3 n-1$ which satisfies

$$
H_{n}^{(t)}\left(f, x_{k}\right)=f^{(t)}\left(x_{k}\right), t=0,1,2, k=1,2, \cdots, n
$$

Then the Hermite interpolation polynomial is given by

$$
H_{n}(f, x)=\sum_{k=1}^{n} f\left(x_{k}\right) L_{k, 0}(x)+\sum_{k=1}^{n} f^{\prime}\left(x_{k}\right) L_{k, 1}(x)+\sum_{k=1}^{n} f^{\prime \prime}\left(x_{k}\right) L_{k, 2}(x)
$$

where

$$
\begin{gathered}
L_{k, 0}(x)=l_{k}^{3}(x)-\frac{9 x_{k}}{2\left(1-x_{k}^{2}\right)}\left(x-x_{k}\right) l_{k}^{3}(x)+\frac{1}{2}\left(\frac{12 x_{k}^{2}}{\left(1-x_{k}^{2}\right)^{2}}+\frac{n^{2}+2 n-3}{1-x_{k}^{2}}\right)\left(x-x_{k}\right)^{2} l_{k}^{3}(x) \\
L_{k, 1}(x)=\left(x-x_{k}\right) l_{k}^{3}(x)-\frac{9 x_{k}}{2\left(1-x_{k}^{2}\right)}\left(x-x_{k}\right)^{2} l_{k}^{3}(x) \\
L_{k, 2}(x)=\frac{\left(x-x_{k}\right)^{2}}{2} l_{k}^{3}(x) \\
l_{k}(x)=\frac{U_{n}(x)}{U_{n}^{\prime}(x)\left(x-x_{k}\right)}=\frac{(-1)^{k}\left(1-x_{k}^{2}\right) U_{n}(x)}{(n+1)\left(x-x_{k}\right)}
\end{gathered}
$$




\section{Theorem 1.}

Let $H_{n}(f, x)$ be defined as (1.1), for $f \in C_{[-1,1]}^{2}$ and $p>0, \alpha>-1$, then we have

$$
\int_{-1}^{1}\left|H_{n}^{\prime}(f, x)-f^{\prime}(x)\right|^{p}\left(1-x^{2}\right)^{\alpha} \mathrm{d} x \leq \begin{cases}C n^{p-2 \alpha-2} E_{3 n-3}^{p}\left(f^{\prime \prime}\right), & p-2 \alpha-1>1 \\ C \ln n E_{3 n-3}^{p}\left(f^{\prime \prime}\right), & p-2 \alpha-1=1 .\end{cases}
$$

\section{Some Lemmas}

Lemmas 1. [6] Let $H_{n}(f, x)$ be defined as (1.1), then

$$
L_{k, h}(x)=\frac{A(x)}{\left(x-x_{k}\right)^{\alpha_{k}}} \cdot \frac{\left(x-x_{k}\right)^{h}}{h !} \cdot\left\{\frac{\left(x-x_{k}\right)^{\alpha_{k}}}{A(x)}\right\}_{\left(x-x_{k}\right)}^{\left(\alpha_{k}-h-1\right)}
$$

where $A(x)=\prod_{k=1}^{n}\left(x-x_{k}\right)^{\alpha_{k}}, \alpha_{k} \in N, \quad \alpha_{1}+\alpha_{2}+\cdots+\alpha_{n}=m+1,\left\{\frac{\left(x-x_{k}\right)^{\alpha_{k}}}{A(x)}\right\}_{\left(x-x_{k}\right)}^{\left(\alpha_{k}-h-1\right)}$ is defined as function $\frac{\left(x-x_{k}\right)^{\alpha_{k}}}{A(x)}$ at $x=x_{k}$ before the commencement of the Taylor series of $\alpha_{k}-h$.

Lemma 2. [7]

If $f \in C_{[-1,1]}^{2}$, then there exists a algebraic polynomial $p_{3 n-1}(x)$ of degree at most $3 n-1$ such that

$$
\left|f^{(i)}(x)-p_{3 n-1}^{(i)}(x)\right| \leq C\left[\frac{\sqrt{1-x^{2}}}{n}\right]^{2-i} E_{3 n-2}\left(f^{\prime \prime}\right) . \quad i=0,1,2
$$

Let

$$
-1=t_{2 n}<t_{2 n-1}<\cdots<t_{1}<t_{0}=1
$$

be the zeros of $\left(1-x^{2}\right) U_{2 n-1}(x)$, here $U_{n-1}(x)=\frac{\sin n \theta}{\sin \theta}, x=\cos \theta$, the $n$th degree Chebyshev polynomial of the second kind. For $f \in C_{[-1,1]}^{1}$, the well-known Lagrange interpolation polynomial of $f$ based on $\left\{t_{k}\right\}_{k=0}^{2 n}$ is given by

$$
Q_{2 n}(f, x)=\sum_{k=0}^{2 n} f\left(t_{k}\right) \varphi_{k}(x)
$$

where

$$
\begin{gathered}
\varphi_{0}(x)=\frac{(1+x) U_{2 n-1}(x)}{2 U_{2 n-1}(1)} \\
\varphi_{2 n}(x)=\frac{(1-x) U_{2 n-1}(x)}{2 U_{2 n-1}(-1)} \\
\varphi_{k}(x)=\frac{(-1)^{k+1}\left(1-x^{2}\right) U_{2 n-1}(x)}{2 n\left(x-t_{k}\right)}, k=1, \cdots, 2 n-1
\end{gathered}
$$

Lemma 3. [7] Let $\varphi_{k}(x), k=0,1, \cdots, 2 n$ be defined as (2.4), for $\alpha, \beta>-1$, and $p>0$, we have

$$
\left(\int_{-1}^{1}\left|\sum_{k=1}^{2 n-1} A_{k} \varphi_{k}(x)\right|^{p}(1-x)^{\alpha}(1+x)^{\beta} \mathrm{d} x\right)^{\frac{1}{p}} \leq C \max _{1 \leq k \leq 2 n-1}\left|A_{k}\right| .
$$




\section{The Proof of Theorem 1}

For $f \in C_{[-1,1]}^{2}$, let $p_{3 n-1}(x)$ be the polynomial of degree at most $3 n-1$ which satisfies Lemma 2 . By the uniqueness of Hemite interpolation polynomial, it can be easily checked that,

$$
H_{n}^{\prime}(f, x)-f^{\prime}(x)=H_{n}^{\prime}\left(f-p_{3 n-1}, x\right)+p_{3 n-1}^{\prime}(x)-f^{\prime}(x)
$$

We can conclude that

$$
\begin{aligned}
& I=\int_{-1}^{1}\left|H_{n}^{\prime}(f, x)-f^{\prime}(x)\right|^{p}\left(1-x^{2}\right)^{\alpha} \mathrm{d} x \\
& \leq 2^{p} \int_{-1}^{1}\left|H_{n}^{\prime}\left(f-p_{3 n-1}, x\right)\right|^{p}\left(1-x^{2}\right)^{\alpha} \mathrm{d} x+2^{p} \int_{-1}^{1}\left|p_{3 n-1}^{\prime}\left(x_{k}\right)-f^{\prime}\left(x_{k}\right)\right|^{p}\left(1-x^{2}\right)^{\alpha} \mathrm{d} x \\
& =2^{p}\left(I_{1}+I_{2}\right) .
\end{aligned}
$$

Firstly, we estimate $I_{1}$. By (3.1), we have

$$
\begin{aligned}
I_{1} \leq & 3^{p} \int_{-1}^{1}\left|\sum_{k=1}^{n}\left(f\left(x_{k}\right)-P_{3 n-1}\left(x_{k}\right)\right) L_{k, 0}^{\prime}(x)\right|^{p}\left(1-x^{2}\right)^{\alpha} \mathrm{d} x \\
& +3^{p} \int_{-1}^{1}\left|\sum_{k=1}^{n}\left(f^{\prime}\left(x_{k}\right)-P_{3 n-1}^{\prime}\left(x_{k}\right)\right) L_{k, 1}^{\prime}(x)\right|^{p}\left(1-x^{2}\right)^{\alpha} \mathrm{d} x \\
& +3^{p} \int_{-1}^{1}\left|\sum_{k=1}^{n}\left(f^{\prime \prime}\left(x_{k}\right)-P_{3 n-1}^{\prime \prime}\left(x_{k}\right)\right) L_{k, 2}^{\prime}(x)\right|^{p}\left(1-x^{2}\right)^{\alpha} \mathrm{d} x \\
= & 3^{p}\left(I_{11}+I_{12}+I_{13}\right) .
\end{aligned}
$$

Firstly, we estimate $I_{11}$,

$$
\begin{aligned}
I_{11} \leq & 3^{p} \int_{-1}^{1} \mid \sum_{k=1}^{n}\left(f\left(x_{k}\right)-P_{3 n-1}\left(x_{k}\right)\right)\left(3 l_{k}^{2}(x) l_{k}^{\prime}(x)-\frac{9 x_{k}}{2\left(1-x_{k}^{2}\right)} l_{k}^{3}(x)||^{p}\left(1-x^{2}\right)^{\alpha} \mathrm{d} x\right. \\
& +3^{p} \int_{-1}^{1} \mid \sum_{k=1}^{n}\left(f\left(x_{k}\right)-P_{3 n-1}\left(x_{k}\right)\right)\left(-\frac{27 x_{k}}{2\left(1-x_{k}^{2}\right)}\left(x-x_{k}\right) l_{k}^{2}(x) l_{k}^{\prime}(x)\right. \\
& +\frac{3}{2}\left(\frac{12 x_{k}^{2}}{\left(1-x_{k}^{2}\right)^{2}}+\frac{n^{2}+2 n-3}{1-x_{k}^{2}}\right)\left(x-x_{k}\right)^{2} l_{k}^{2}(x) l_{k}^{\prime}(x) \mid\left(1-x^{2}\right)^{\alpha} \mathrm{d} x \\
& +3^{p} \int_{-1}^{1}\left|\sum_{k=1}^{n}\left(f\left(x_{k}\right)-P_{3 n-1}\left(x_{k}\right)\right)\left(\frac{12 x_{k}^{2}}{\left(1-x_{k}^{2}\right)^{2}}+\frac{n^{2}+2 n-3}{1-x_{k}^{2}}\right)\left(x-x_{k}\right) l_{k}^{3}(x)\right|^{p}\left(1-x^{2}\right)^{\alpha} \mathrm{d} x \\
= & 3^{p}\left(I_{A}+I_{B}+I_{C}\right) .
\end{aligned}
$$

Let

$$
B(x)=\sum_{k=1}^{n}\left(f\left(x_{k}\right)-P_{3 n-1}\left(x_{k}\right)\right)(-1)^{k}\left(1-x_{k}^{2}\right) U_{n}(x)\left(\frac{l_{k}(x) l_{k}^{\prime}(x)}{\left(x-x_{k}\right)}-\frac{3 x_{k} l_{k}^{2}(x)}{2\left(1-x_{k}^{2}\right)\left(x-x_{k}\right)}\right)
$$

be the polynomial of degree $2 n-3$. By the uniqueness of Lagrange interpolation polynomial, it can be easily checked that,

$$
B(x)=\sum_{l=0}^{2 n} B\left(t_{l}\right) \varphi_{l}(x)
$$

By (3.5), (3.6) and Lemma 3, we can derive 


$$
\begin{aligned}
I_{A} \leq & \frac{C}{(n+1)^{p}}\left(\max _{0 \leq l \leq 2 n-1}\left|\sum_{k=1}^{n}\left(f\left(x_{k}\right)-P_{3 n-1}\left(x_{k}\right)\right)(-1)^{k}\left(1-x_{k}^{2}\right) U_{n}\left(t_{l}\right)\left(\frac{l_{k}\left(t_{l}\right) l_{k}^{\prime}\left(t_{l}\right)}{\left(t_{l}-x_{k}\right)}-\frac{3 x_{k} l_{k}^{2}\left(t_{l}\right)}{2\left(1-x_{k}^{2}\right)\left(t_{l}-x_{k}\right)}\right)\right|\right)^{p} \\
& +\frac{3^{2 p}}{(n+1)^{p}} \int_{-1}^{1}\left|\sum_{k=1}^{n}\left(f\left(x_{k}\right)-P_{3 n-1}\left(x_{k}\right)\right)(-1)^{k}\left(1-x_{k}^{2}\right) U_{n}(x)\left(\frac{l_{k}(1) l_{k}^{\prime}(1)}{\left(1-x_{k}\right)}-\frac{3 x_{k} l_{k}^{2}(1)}{2\left(1-x_{k}^{2}\right)\left(1-x_{k}\right)}\right)\right| \\
& \cdot\left|\frac{(1+x) U_{2 n-1}(x)}{2 U_{2 n-1}(1)}\right|^{p}\left(1-x^{2}\right)^{\alpha} \mathrm{d} x \\
& +\frac{3^{2 p}}{(n+1)^{p}} \int_{-1}^{1}\left|\sum_{k=1}^{n}\left(f\left(x_{k}\right)-P_{3 n-1}\left(x_{k}\right)\right)(-1)^{k}\left(1-x_{k}^{2}\right) U_{n}(x)\left(\frac{l_{k}(-1) l_{k}^{\prime}(-1)}{\left(-1-x_{k}\right)}+\frac{3 x_{k} l_{k}^{2}(-1)}{2\left(1-x_{k}^{2}\right)\left(1+x_{k}\right)}\right)\right|^{p} \\
& \cdot\left|\frac{(1+x) U_{2 n-1}(x)}{2 U_{2 n-1}(-1)}\right|^{p}\left(1-x^{2}\right)^{\alpha} \mathrm{d} x \\
= & M_{1}+3^{2 p}\left(M_{2}+M_{3}\right) .
\end{aligned}
$$

Firstly, we estimate $M_{1}$. Let

$$
A(l)=\left|\sum_{k=1}^{n}\left(f\left(x_{k}\right)-P_{3 n-1}\left(x_{k}\right)\right)(-1)^{k}\left(1-x_{k}^{2}\right) U_{n}(x)\left(\frac{l_{k}(x) l_{k}^{\prime}(x)}{\left(x-x_{k}\right)}-\frac{3 x_{k} l_{k}^{2}(x)}{2\left(1-x_{k}^{2}\right)\left(x-x_{k}\right)}\right)\right|
$$

then

$$
l_{k}^{\prime}\left(t_{l}\right)= \begin{cases}\frac{3 x_{k}}{2\left(1-x_{k}^{2}\right)}, & l=2 s, s=k ; \\ \frac{(-1)^{s+k}\left(1-x_{k}^{2}\right)}{\left(1-t_{l}^{2}\right)\left(t_{l}-x_{k}\right)}, & l=2 s, s \neq k ; \\ \frac{(-1)^{s+k+1}\left(1-x_{k}^{2}\right)}{n+1} \cdot \frac{t_{l}}{\left(t_{l}-x_{k}\right)^{2}\left(1-t_{l}^{2}\right)^{\frac{3}{2}}}, & l=2 s-1 .\end{cases}
$$

From Lemma 2 and (3.8), (3.9), we have that for $l=2 s-1$.

$$
A(l) \leq C E_{3 n-3}\left(f^{\prime \prime}\right)
$$

For $l=2 s, s=1,2, \cdots, n$, we have

$$
A(l)=0
$$

We can conclude

$$
M_{1} \leq C E_{3 n-3}^{p}\left(f^{\prime \prime}\right) .
$$

Secondly, we estimate $M_{2}$, and by Lemma 2, we get

$$
M_{2} \leq \begin{cases}C n^{p-2 \alpha-2} E_{3 n-3}^{p}\left(f^{\prime \prime}\right) & p-2 \alpha-1>1 \\ C \ln n E_{3 n-3}^{p}\left(f^{\prime \prime}\right) & p-2 \alpha-1=1\end{cases}
$$

Similarly

$$
M_{3} \leq \begin{cases}C n^{p-2 \alpha-2} E_{3 n-3}^{p}\left(f^{\prime \prime}\right) & p-2 \alpha-1>1 \\ C \ln n E_{3 n-3}^{p}\left(f^{\prime \prime}\right) & p-2 \alpha-1=1\end{cases}
$$


By (3.12), (3.13) and (3.14), we have

$$
I_{A} \leq \begin{cases}C n^{p-2 \alpha-2} E_{3 n-3}^{p}\left(f^{\prime \prime}\right) & p-2 \alpha-1>1 \\ C \ln n E_{3 n-3}^{p}\left(f^{\prime \prime}\right) & p-2 \alpha-1=1\end{cases}
$$

Similarly, we get

$$
\begin{gathered}
I_{B} \leq \begin{cases}C n^{p-2 \alpha-2} E_{3 n-3}^{p}\left(f^{\prime \prime}\right) & p-2 \alpha-1>1 \\
C \ln n E_{3 n-3}^{p}\left(f^{\prime \prime}\right) & p-2 \alpha-1=1\end{cases} \\
I_{C} \leq \begin{cases}C n^{p-2 \alpha-2} E_{3 n-3}^{p}\left(f^{\prime \prime}\right) & p-2 \alpha-1>1 \\
C \ln n E_{3 n-3}^{p}\left(f^{\prime \prime}\right) & p-2 \alpha-1=1\end{cases}
\end{gathered}
$$

By (3.15), (3.16) and (3.17), we get

$$
I_{11} \leq \begin{cases}C n^{p-2 \alpha-2} E_{3 n-3}^{p}\left(f^{\prime \prime}\right) & p-2 \alpha-1>1 \\ C \ln n E_{3 n-3}^{p}\left(f^{\prime \prime}\right) & p-2 \alpha-1=1\end{cases}
$$

Similarly, we get

$$
\begin{aligned}
& I_{12} \leq C E_{3 n-3}^{p}\left(f^{\prime \prime}\right) . \\
& I_{13} \leq C E_{3 n-3}^{p}\left(f^{\prime \prime}\right) .
\end{aligned}
$$

Secondly, we estimate $I_{2}$, from Lemma 2,

$$
I_{2} \leq C E_{3 n-3}^{p}\left(f^{\prime \prime}\right) .
$$

From (3.2), (3.3), and (3.21), we can obtain the upper estimate

$$
I \leq \begin{cases}C n^{p-2 \alpha-2} E_{3 n-3}^{p}\left(f^{\prime \prime}\right) & p-2 \alpha-1>1 \\ C \ln n E_{3 n-3}^{p}\left(f^{\prime \prime}\right) & p-2 \alpha-1=1\end{cases}
$$

\section{Funding}

Supported by Educational Commission of Hebei Province of China (NO. QN20132001).

\section{References}

[1] Szabados, J. and Vestesi, P. (1992) A Survey on Mean Convergence of Interpolatory Processes. Journal of Computational and Applied Mathematics, 43, 3-18. http://dx.doi.org/10.1016/0377-0427(92)90256-W

[2] Szabados, J. and Vertesi, P. (1990) Interpolation of Functions. World Scientific, Singapore.

[3] Wang, X. (2011) The Approximation of Hermite Interpolation on the Weighted Mean Norm. Journal of Tianjin Normal University, 31, 7-12.

[4] Xia, Y. and Xu, G.Q. (2010) The Approximation of Hermite Interpolation on the Weighted Mean Norm. Journal of Tianjin Normal University, 31, 6-10.

[5] Xu, G.Q., Cui, R. and Wang, X. (2009) The Simultaneous Approximation of Quasi-Hermite Interpolation on the Weighted Mean Norm. International Journal of Wavelets, Multiresolution and Information Processing, 7, 825-837. http://dx.doi.org/10.1142/S0219691309003276

[6] Xie, T.F. and Zhou, S.P. (1998) Real Function Approximation Theory. Hangzhou University Press, Hangzhou.

[7] Kingore, T. (1993) An Elementary Simultaneous Polynomials. Proceedings of the American Mathematical Society, 118, 529-536. http://dx.doi.org/10.1090/S0002-9939-1993-1129881-X 